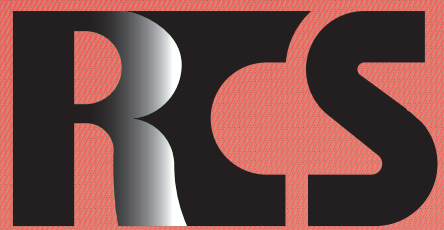

Depósito legal ppi $201502 Z U 4662$

Esta publicación científica en formato digital es continuidad de la revista im Depósito Legal: pp $197402 Z U 789$

- ISSN: 1315-9518 • ISSN-E: 2477-'

Revista de Ciencias Sociales

Universidad del Zulia. Revista de la Facultad de Ciencias Económicas y Sociales Vol. XXVII. No. 2

Abril-Junio 2021

Esta publicación científica en formato digital es continuidad de la revista impresa Depósito Legal: pp $197402 Z$ Z789 ISSN: 1315-9518 


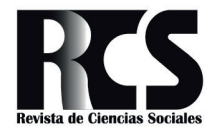

Revista de Ciencias Sociales (RCS). FCES - LUZ

Vol. XXVII, No. 2, 2021, pp. 8-11 Abril-Junio

- ISSN: 1315-9518 ISSN-E: 2477-9431

Editorial

\section{Un Jardín de ideas y conocimientos florece en tiempos de tempestad, ofreciendo un contraste armonioso de alternativas a la sociedad para afrontar las vicisitudes}

En un contexto tormentoso donde predominan crisis económicas, sanitarias, sociales, políticas, ambientales y religiosas, entre otras, las sociedades a nivel global deben afrontar de manera impositiva, todavía como consecuencia del Covid-19, cambios permanentes en procura de una estabilidad emocional, de salud y socioeconómica, donde al mismo tiempo se han desdibujado perfiles laborales, conduciendo a reajustes de prácticas sociales, empresariales, gubernamentales, profesionales -sobre todo en el campo educativo y social-, para afrontar una realidad con importantes dilemas.

Ante ello, la Revista de Ciencias Sociales (Ve), en este volumen XXVII, No. 2-2021, ofrece un jardín de múltiples ideas desde una amalgama de coloridos espacios, que en su conjunto armonioso, el ser humano ha empleado y puede seguir empleando, para superar vicisitudes y sembrar nuevos jardines o reacondicionar los existentes con el fin de continuar creando conocimientos, reflexionar y seguir investigando en un entorno desafiante que requiere florecimiento de ideas, con mayor amplitud y alcances interdisciplinarios.

En un marco de tempestad, esta revista sigue prosperando con el pensamiento de los autores y esfuerzos de la editora, equipo editorial, comité científico y evaluadores, entre otros, con grandes retos, pero sobretodo con la responsabilidad de demostrar las capacidades para compartir los acontecimientos inéditos, que ha evocado a hacer ciencia ante una normalidad donde se conjugan momentos de lluviosos temores y otros resplandecientes, cargados de energía y senderos de luz; y así, entre temores y energías, la Revista de Ciencias Sociales presenta a los investigadores y estudiosos que hicieron frente con sus habilidades e inteligencia a los procesos sociales y nuevos fenómenos que han surgidos en las sociedades, para abonar el jardín de ideas y conocimientos, así como compartir los acontecimientos en la realidad social.

Sin dejar de lado que las dificultades aun persistentes, también han emanado iniciativas sociales e institucionales, orientadas a promover los valores en la sociedad, así como la importancia de la ciencia y de su influencia en la salud, educación, alimentación y conservación del medio ambiente, exigiendo tanto a universidades, laboratorios, equipos de investigación, como a entidades científicas, a evolucionar con capacidad crítica y creativa, de acuerdo con las 
demandas progresivas de los seres humanos, pero a la vez, avanzar con mecanismos y estrategias multidisciplinarias para contribuir a la solución de problemas sociales crecientes y difíciles de resolver.

Como de costumbre, los autores de este número desarrollan sus investigaciones desde prestigiosas instituciones y universidades nacionales e internacionales, a las cuales se les agradece sus colaboraciones y empeño en el devenir investigativo, entre las cuales en esta oportunidad acompañan desde España, la Universidad de Jaén, Universidad de Sevilla y la Universidad Internacional de la Rioja. También trabajos provenientes de México, destacan la Universidad Autónoma del Estado de Hidalgo, Universidad de Sonora y la Universidad Autónoma de Guerrero. Desde el mismo continente latinoamericano, autores chilenos desarrollaron sus artículos en la Universidad Andrés Bello, Universidad de Tarapacá, Universidad de Concepción y la Universidad Austral de Chile. Ecuador, también comparte estudios que surgieron de la Universidad Técnica de Machala, Instituto Superior Tecnológico El Oro - Machala y la Universidad Técnica de Manabí.

En la cercanía geográfica a Venezuela, Colombia tuvo una presencia contundente (diecisiete entidades), a saber: Universidad de San Buenaventura, Academia Colombiana de Historia, Universidad de Manizales, Universidad Corporación Reformada de Barranquilla, Universidad de la Costa, Tecnológico de Antioquia, Universidad Libre, Instituto Tecnológico de Soledad Atlántico, Corporación Universitaria Latinoamericana, Universidad Santiago de Cali, Universidad del Atlántico, Instituto Colombiano de Estudios Superiores de Incolda, Corporación Universitaria Autónoma de Nariño, Fundación Universitaria de Popayán, Corporación Universitaria Latinoamericana, Tecnológico de Antioquia y la Universidad de la Amazonia.

También en este número hizo acto de presencia Perú, con trabajos que se originaron en la Universidad Nacional del Altiplano, Universidad Católica de Santa María, Universidad César Vallejo, Universidad Señor de Sipán, Universidad Privada del Norte, Universidad Nacional de San Agustín de Arequipa y la Universidad Privada del Norte; y en el ámbito nacional (Venezuela), la Universidad Dr. Rafael Belloso Chacín y la propia casa de estudios: Universidad del Zulia.

De igual forma, se puede observar en esta revista la variedad de temas que han sido impactados por la pandemia y siguen siendo afectados por la misma en todos los países del planeta, acentuado el compromiso con las comunidades de dotarlas de resultados de investigación que aporten a la conservación, desarrollo y difusión del conocimiento, en concordancia con las exigencias internacionales, garantizando la cientificidad y calidad de los trabajos aquí publicados.

En esta oportunidad, los lectores podrán acceder a una nota de actualidad, un ensayo y treinta y un artículos científicos, que giran alrededor de diversidad de temáticas de ciencias sociales. Así, en la nota de actualidad, se aborda la innovación social y ciencia ciudadana en la gestión patrimonial, destacando la necesidad de profesionalización y apertura social en el desarrollo comunitario. Por su parte, el ensayo trata de problemáticas éticas, ontológicas y jurídicas que se derivan de la inteligencia artificial en las sociedades actuales. Con respecto a los artículos científicos seis trabajos en el ámbito de las ciencias económicas con diferentes vertientes es un interesante abreboca de este número, entre ellos se encuentran: Desarrollo sostenible del oleoturismo: Un análisis de la colaboración entre actores; Desarrollo local en el municipio de Corinto en CaucaColombia en el marco del posconflicto; Economía social como alternativa ante una sociedad post coronavirus; Modelo logístico para el aprovechamiento del potencial turístico del municipio Santo Tomás-Atlántico en Colombia; Mestizaje, creolización, sincretismo e hibridación cultural, a través de los mercados populares en América; $y$, Situación de la mediana empresa ecuatoriana: Rasgos distintivos de sus prácticas gerenciales.

En las ciencias sociales, la educación generalmente es una temática del interés de los investigadores y así se evidencia en los siete estudios que se esbozan desde estrategias pedagógicas, competencias genéricas, formación, emprendimiento universitario, gestión universitaria, responsabilidad social universitaria e inclusión social, sobre esos aspectos, se 
Editorial.

Un Jardin de ideas y conocimientos florece en tiempos de tempestad, ofreciendo un contraste armonioso de alternativas a la sociedad para afrontar las vicisitudes.

María Cristina Useche Aguirre

encuentran los siguientes trabajos: Estrategias pedagógicas para el aprendizaje y desarrollo de competencias investigativas en estudiantes universitarios; Plan de acompañamiento académico: Incidencia en el desarrollo de competencias genéricas en estudiantes universitarios; COVID-19, educación en emprendimiento e intenciones de emprender: Factores decisorios en estudiantes universitarios; Formación del profesorado en Latinoamérica; Proceso vital en la gestión educativa: Herramienta de alta calidad hacia la sostenibilidad ambiental; Innovación social: ¿Nueva cara de la responsabilidad social? conceptualización crítica desde la perspectiva universitaria; y, la Inclusión social de la población estudiantil afrodescendiente: Experiencia de un colectivo de estudiantes universitarios.

El enfoque de género en las últimas décadas ha tomado gran impulso y desde el año 2020 países latinoamericanos y la CEPAL han declarado una involución en la igualdad de género; al respecto, tres investigaciones emanan gran complejidad por insertar los derechos, la pobreza y el ruralismo en sus temáticas, así disfrutarán de: Mujeres no son personas: Los derechos de las mujeres nahuas de Acaxochitlán, Hidalgo-México; Masculinidad, migración y pobreza extrema: Mirada retrospectiva de exmigrantes en Hermosillo, Sonora-México y Turismo rural en CrucitaEcuador: Una mirada desde la fortaleza del género.

El ámbito empresarial en el siglo XXI, siempre ha ampliado los recursos en investigaciones sobre gestión, eficiencia de sus procesos, desempeño laboral, liderazgo y formación del talento humano, y así se deja ver en los trabajos referentes a: Hacia la responsabilidad social como estrategia de sostenibilidad en la gestión empresarial; Proceso administrativo y sostenibilidad empresarial del sector hotelero de la parroquia Crucita, ManabiEcuador; Compromiso organizacional y factores demográficos que propician el ausentismo laboral en franquicias de Barranquilla-Colombia; Liderazgo ético en la gestión pública municipal del estado Zulia, Venezuela; Liderazgo como prospectiva del clima organizacional en el sector hotelero; Formación por competencias del profesional en administración: Desde un enfoque contingencial; $y$, Determinantes del desempeño gerencial: Instituciones de Educación Superior del departamento Atlántico-Colombia frente al Covid-19.

El lector, también encontrará en el turismo un estudio que destaca el cuidado del medio ambiente referente al: Potencial paisajístico de la Laguna de Coyuca de Benitez: Detonante de productos sustentables en Acapulco-México. Por otra parte, un trabajo histórico de Maracaibo puede consultarse en: Capitulación de Maracaibo: Revisiones historiográficas de la Independencia desde la perspectiva de la paz, en el cual, se demuestra el aporte en el proceso de liberación de Venezuela. Este trabajo, se relaciona con el siguiente en que ambos consideran los conocimientos histórico; sin embargo, en el posterior estudio titulado: Tradición pesquera artesanal e identidad sociocultural de Puerto Bolivar: Contexto del Golfo de GuayaquilEcuador, destacan el arraigo más cultural que económico en ese país.

La precariedad laboral siempre ha sido un tema de discusión en las ciencias sociales, que se ha acentuado con el COVID-19, como lo ha demostrado la Organización Internacional del Trabajo; por lo que, resulta oportuno la investigación que se realizara en Colombia en un contexto del conflicto armado, donde existen vulnerabilidad en la comunidad afrodescendiente y los pueblos indígenas, intitulado: Precariedad laboral en población afrodescendiente e indígena agravada por el conflicto armado en Colombia. Siguiendo en el ámbito laboral, pero desde otra corriente, estudios han demostrado que jóvenes no tutelados tienen altas tasas de desempleo, acerca de esto versa el artículo sobre: Programa de transición a la vida adulta en Andalucía: Percepción de profesionales y extutelados, y aunque el programa genera grandes beneficios a los jóvenes, es necesario mejorar y aumentar subvenciones así como concientizar a la población en cuanto a la importancia de estos programas.

Sobre tres temas distintos pero de gran pertinencia como el clima, inmigración y el urbanismo, se quieren hacer mención, pues también fueron tratados en este número, el 
siguiente artículo busca: Reconocer a la bestia: Percepción de peligro climático en estudiantes de educación secundaria, y consideran que es necesario reforzar los modelos pedagógicos e institucionalizar la transversalización del cambio climático en Perú. En relación al trabajo: Propiedades psicométricas de la escala de amenaza grupal internalizada por inmigrantes latinoamericanos en Chile, tiene interesantes resultados basados en la teoría de la amenaza y el prejuicio en inmigrantes latinoamericanos; y finalmente, el estudio acerca de la Planificación territorial en Chile: Del modelo Top Down a los desafios de articulación multinivel, se destaca que el desarrollo urbano ha tenido avances importantes en Chile; sin embargo, la práctica generalizada limita su expansión.

Finalmente, se desea que este número sea de interés y utilidad para todas las entidades investigativas nacionales e internacionales, confiando en que los artículos, nota y ensayo generen aportes e impactos positivos a las diferentes sociedades. En tal sentido, se agradece a cada uno de los autores por sus participaciones y se invita al lector a revisar con cautela las diversas secciones, al tiempo que se espera poder seguir cuidando, difundiendo y ampliando los jardines de ideas y conocimientos en el futuro.

Dra. María Cristina Useche Aguirre Investigadora-Profesora titular de la Universidad del Zulia (Venezuela) E-mail: mariacristina.useche@,fces.luz.edu.ve ORCID: https://orcid.org/0000-0001-5057-0034 